\title{
GROUPS WITH INFINITE PRODUCTS
}

\author{
BY JOHN STALLINGS ${ }^{1}$
}

Communicated by Deane Montgomery, April 8, 1962

If $G$ is a group, then an infinite product on $G$ is a function $\mu: G^{\infty} \rightarrow H$, where $G^{\infty}$ is the set of sequences of elements of $G$, and $H$ is some other set. I call $\mu$ associative if it satisfies all the associative laws of the form

$$
\begin{aligned}
& \mu\left(x_{1}, x_{2}, \cdots, x_{n}, \cdots\right) \\
& \quad=\mu\left(x_{1} x_{2} \cdots x_{i_{2}-1}, x_{i_{2}} \cdots x_{i_{3}-1}, \cdots, x_{i_{n}} \cdots x_{i_{n+1}-1}, \cdots\right) .
\end{aligned}
$$

Here juxtaposition denotes multiplication in $G$. Then the utter triviality of $\mu$ follows from this trick:

$$
\begin{aligned}
& x_{1} x_{2} x_{3} \cdots \\
& \quad=\left(x_{1} \bar{x}_{1} x_{1}\right)\left(x_{2} \bar{x}_{2} \bar{x}_{1} x_{1} x_{2}\right)\left(x_{3} \bar{x}_{3} \bar{x}_{2} \bar{x}_{1} x_{1} x_{2} x_{3}\right) \cdots \\
& \quad=\left(x_{1} \bar{x}_{1}\right)\left(x_{1} x_{2} \bar{x}_{2} \bar{x}_{1}\right)\left(x_{1} x_{2} x_{3} \bar{x}_{3} \bar{x}_{2} \bar{x}_{1}\right) \cdots \\
& \quad=1 \cdot 1 \cdot 1 \cdots
\end{aligned}
$$

Here $\bar{x}_{n}$ denotes the inverse of $x_{n}$, and 1 denotes the identity of $G$.

A form of this trick was noticed and used by B. Mazur [2]. An example of another use is this.

Let $C$ be a compact Hausdorff space. If $\alpha>0$, define $C(\alpha)$ to be the space $C \times[0, \alpha)$ with $C \times 0$ identified to one point 0 . Define $\Sigma$ to be the set of all those functions $f: C(1) \rightarrow C(1)$ which can be extended to $f_{*}: C(2) \rightarrow C(1)$ where $f_{*}$ is a homeomorphism onto an open subset of $C(1)$, such that $f(0)=0$. Define $\Gamma$ to be the set of those homeomorphisms $\phi: C(1) \rightarrow C(1)$ for which there is $\epsilon>0$ such that $\phi$ is the identity on $C(\epsilon) \cup[C(1)-C(1-\epsilon)]$.

If $f$ and $g$ belong to $\Sigma$, define $f \sim g$ to mean there exists $\phi \in \Gamma$ such that $f=g \phi$, where the notation here for composition of maps is that $g \phi(x)=\phi(g(x))$. It can be shown that $f \sim g$ if and only if there is $\epsilon>0$ such that $f|C(\epsilon)=g| C(\epsilon)$.

From this, one can deduce that the equivalence classes of $\Sigma$ under the relation $\sim$ form a group with multiplication induced by the composition of maps; this group will be called $G$.

Now if $f_{1}, f_{2}, \cdots$, is a sequence of elements of $\Sigma$, define $\mu\left(f_{1}, f_{2}, \cdots\right)$ to be the direct limit of the sequence of spaces and maps

$$
C(1) \stackrel{f_{1}}{\rightarrow} C(1) \stackrel{f_{2}}{\rightarrow} C(1) \rightarrow \cdots \cdot
$$

\footnotetext{
1 This research was supported by the Air Force Office of Scientific Research.
} 
One can show that $\mu\left(f_{1}, f_{2}, \cdots\right)$ is determined up to homeomorphism by the equivalence classes of $f_{1}, f_{2}$, etc. The associativity, up to homeomorphism, of $\mu$ is simply the statement that the direct limit of a directed set of spaces and maps is homeomorphic to the direct limit of a cofinal subset.

The associativity trick then proves that the spaces $\mu\left(f_{1}, f_{2}, \cdots\right)$ are all homeomorphic to each other; a particular such space can be shown homeomorphic to $C(\infty)$ or $C(1)$.

It follows from the compactness of $C$, that if $X$ is a space which is the union of its open subsets $U_{n}$, each of which is homeomorphic to $C(\infty)$ in such a way that the odd points 0 coincide for all $n$, and if every compact subset of $X$ is contained in some $U_{n}$, then $X$ is homeomorphic to some space of the form $\mu\left(f_{1}, f_{2}, \cdots\right)$. And hence $X$ is homeomorphic to $C(\infty)$.

Taking $C$ to be the $(n-1)$-sphere, one obtains the theorem of $\mathrm{M}$. Brown [1] that a monotone union of open $n$-cells is an open $n$-cell.

This is perhaps the most conceptual way to understand my proof [3] of several generalizations of Brown's theorem, although if written out in detail this method would be no shorter.

\section{REFERENCES}

1. M. Brown, The monotone union of open n-cells is an open n-cell, Proc. Amer. Math. Soc. 12 (1961), 812-814.

2. B. Mazur, On embeddings of spheres, Bull. Amer. Math. Soc. 65 (1959), 59-65.

3. J. Stallings, On a theorem of Brown about the union of open cones, Ann. of Math. (to appear).

Princeton University AND

The Institute for Advanced Study 